(C) 2021 IEEE. Personal use of this material is permitted. Permission from IEEE must be obtained for all other uses, in any current or future media, including reprinting/republishing this material for advertising or promotional purposes, creating new collective works, for resale or redistribution to servers or lists, or reuse of any copyrighted component of this work in other works

The version of record is available at https://doi.org/10.1109/IC3D53758.2021.9687222 


\title{
THE PERCEPTUALLY-SUPPORTED AND THE SUBJECTIVELY-PREFERRED VIEWING DISTANCE OF PROJECTION-BASED LIGHT FIELD DISPLAYS
}

\author{
Peter A. Kara ${ }^{a, b}$, Mary Guindy ${ }^{c, d}$, Tibor Balogh ${ }^{c}$, Aniko Simon \\ ${ }^{a}$ Department of Networked Systems and Services, \\ Budapest University of Technology and Economics, Budapest, Hungary \\ Email: kara@hit.bme.hu \\ ${ }^{\mathrm{b}}$ Kingston University, London, UK \\ Email: p.kara@kingston.ac.uk \\ ${ }^{\mathrm{c}}$ Holografika, Budapest, Hungary \\ Email: \{m.guindy, t.balogh\}@holografika.com \\ ${ }^{d}$ Pazmany Peter Catholic University, Budapest, Hungary \\ Email: guindy.mary.mohsen.messak@ppke.hu \\ e Sigma Technology, Budapest, Hungary \\ Email: aniko.simon@sigmatechnology.se
}

\begin{abstract}
As the research efforts and development processes behind light field visualization technologies advance, potential novel use cases emerge. These contexts of light field display utilization fundamentally depend on the distance of observation, due to the sheer technological nature of such glasses-free 3D systems. Yet, at the time of this paper, the number of works in the scientific literature that address viewing distance is rather limited, focusing solely on 3D visual experience based on angular density. Thus far, the personal preference of observers regarding viewing distance has not been considered by studies. Furthermore, the upcoming standardization efforts also necessitate research on the topic in order to coherently unify the methodologies of subjective tests. In this paper, we investigate the perceptually-supported and the subjectively-preferred viewing distance of light field visualization. We carried out a series of tests on multiple projection-based light field displays to study these distances, with the separate involvement of experts and regular test participants.
\end{abstract}

Index Terms - Light field, 3D content, viewing distance, user preference.

\footnotetext{
The scientific efforts leading to the results reported in this paper were supported by the National Research Development and Innovation Fund based on the charter of bolster issued by the National Research Development and Innovation Office under the auspices of the Ministry for Innovation and Technology, Hungary; the European Union's Horizon 2020 research and innovation programme under the Marie Skłodowska-Curie grant agreement No 813170; 2018-2.1.3-EUREKA-2018-00007 and KFI 16-1-2017-0015, NRDI Fund, Hungary.
}

\section{INTRODUCTION}

Light field technology is progressing rapidly. While the penetration of the consumer market is still a relatively long-term goal, innovation within the scientific community and the industry frequently introduces novel display categories. For example, the very recent work of Balogh et al. [1] presents the prototype of a 3D light field LED wall, which is practically an any-size, any-aspect, any-shape glasses-free 3D display. At the same time, research on projection-based light field visualization is thriving, yet experiments that involve human observers still suffer the lack of standardized test methodologies.

One particular aspect of experimental configurations is the viewing distance of test participants. For conventional 2D displays, this is thoroughly standardized through the resolution of the screen and its height (commonly denoted as $\mathrm{H}$ ), and significant research efforts are continuously improving our understanding of the influence of viewing distance on perceived quality. For example, the work of Amirpour et al. [2] provides an estimation of potential bitrate savings based on the viewing distance - since contents with low media encoding quality levels are perceived to be better at greater distances - and confirms the need for distance-aware objective quality metrics. However, at the time of this paper, there is no standard that covers the viewing distance for the quality assessment of light field visualization, and the topic in general is rather underinvestigated. Particularly, no work so far studied the viewers' personal preference regarding viewing 
distance, and the currently existing literature only addresses the $3 \mathrm{D}$ experience of the viewers [3] but and not the actual perceptual thresholds.

In this paper, we present our work on the perceptuallysupported and the subjectively-preferred viewing distance of light field visualization. We investigated the perceptuallysupported viewing distance through content detail discrimination on a back-projection light field display. The subjectivelypreferred viewing distance was assessed on the same display via content-dependent distance selection. The latter was repeated for a front-projection light field display as well. The experiments were completed by experts and regular test participants, and the data coming from the distinct groups is analyzed and discussed separately. Additionally, as the regular test participants for the two experiments were pooled from the same set of individuals, the correlation between these results is also examined.

The remainder of the paper is structured as follows: Section 2 reviews the relevant scientific literature. Section 3 details the experimental setup of the tests. Section 4 introduces and discusses the obtained results. Section 5 concludes the paper and highlights potential future continuations of the presented research.

\section{RELATED WORK}

As of 2021, there is not a single international standard that addresses the viewing distance of light field visualization. Yet, for nearly a decade now, numerous works have been published, in which test participants assessed different Key Performance Indicators (KPIs) [4] and other vital attributes and contents of this technology. The lack of standardized methodologies led to multiple arbitrary values in the experimental configurations of these works. What is even worse, is that there are quite a few works that do not report the viewing distance at all. Moreover, the majority of those works that do report the chosen viewing distance does not explain the rationale behind the selected value.

In the work of Ahar et al. [5], the authors state that "the distance from the screen was chosen 3.2 times the height of the screen" (which corresponded to $280 \mathrm{~cm}$, as the HoloVizio 722RC light field display $1{ }^{1}$ was used), and that the test participants assessed the stimuli from the center and the right-corner positions. While the methodology of the subjective study followed the Rec. ITU-R BT.500-13 guidelines - the annex of which does specify an H-based approach - the selection of $3.2 \mathrm{H}$ is not explained.

Both Cserkaszky et al. [6], Darukumalli et al. [7] and Kara et al. [8] selected $460 \mathrm{~cm}$ as viewing distance for the quality assessment performed on the HoloVizio C80 light field

\footnotetext{
${ }^{1}$ https://holografika.com/722rc/

${ }^{2}$ https://www.itu.int/dms_pubrec/itu-r/rec/bt/R-REC-BT.500-13-201201I!!PDF-E.pdf
}

cinema system ${ }^{3}$, which is a large-scale horizontal-only parallax (HOP) light field display. While these works do indicate the $\mathrm{H}$-based distance $(2.5 \mathrm{H})$, the reason behind the choice of this viewing distance originated from the physical structure of the display. As the C80 is a front-projection light field display, in order to avoid even the slightest chance of invalid light field, the authors refrained from having test participants closer to the screen of the display than the projector array. In fact, the test participants were positioned directly behind the line of the projector array, hence the $460 \mathrm{~cm}$.

The subjective study of Dricot et al. [9] also used the HoloVizio C80. During the tests, "subjects were sitting at a viewing distance of approximately $6 \mathrm{~m}$ from the screen"; however, the choice of viewing distance is not explained in the publication.

The work of Kawakita et al. [10] presents the assessment of a 200-inch prototype display. The display system design involves a relationship which is used to derive the suitable viewing distance:

$$
\frac{1}{L}+\frac{1}{D}=\frac{1}{f},
$$

where $L$ is the distance between the projectors and the screen, $D$ is the "suitable" viewing distance and $f$ is the focal length. Based on the parameters of the prototype, a viewing distance of $5.5 \mathrm{~m}$ was determined. The authors acknowledge that the ideal gap of parallax images is less than the size of the pupil (i.e., two distinct light rays coming from a single point of the screen can address the pupil), and that the implementation of such system is quite challenging. Furthermore, as this gap is only $22.8 \mathrm{~mm}$, distinct light rays can easily address the two pupils, as the interpupillary distance is commonly measured to be $65 \mathrm{~mm}$. Through these attributes, an additional $2 \mathrm{~m}$ in viewing distance could still be supported in terms of 3D experience.

Lee et al. [11] utilized the lens maker's law in their system design:

$$
\begin{gathered}
d_{v}=\frac{f d_{p}}{d_{p}-f}, \\
p_{e}=\frac{p_{p} d_{v}}{d_{p}},
\end{gathered}
$$

where $d_{v}$ is the "optimal" viewing distance, $f$ is the focal length, $d_{p}$ is the distance between the projectors and the screen, $p_{e}$ is the interval of viewpoints (i.e., analogous to the previously discussed gap, which should be equal to or below the interpupillary distance) and $p_{p}$ is the interval of the adjacent projectors. The viewing distance determined for the system was $1.2 \mathrm{~m}$ while adhering to a viewpoint interval of $65 \mathrm{~mm}$.

\footnotetext{
${ }^{3}$ https://holografika.com/c80-glasses-free-3d-cinema/
} 
The work of Kara et al. [3] uses the rule of thumb in the industry regarding the viewing distance threshold for light field displays:

$$
D_{V}=\frac{D_{E}}{\tan (A R)},
$$

where $D_{V}$ is the viewing distance threshold (i.e., the recommended maximum viewing distance or "the viewing distance at which the 3D experience is still supported"), $D_{E}$ is the interpupillary distance and $A R$ is the angular resolution of the display. The authors carried out a series of subjective tests to assess the practical meaning of this distance. The results clearly indicate that it is the distance at which still, nonmoving observers are unable to determine whether the visual experience is closer to 2D or 3D. In a subsequent work [12], the authors address the use-case-dependent constraints and flexibility of the threshold.

Regarding standardization, while no such standard is published at the time of this paper, the IEEE P3333.1.4 - the Standard for the Quality Assessment of Light Field Imaging - is already approved for development, and it shall directly address viewing distance. The work presented in this paper aims to contribute research data to the standardization efforts. With the guidelines and recommendations of the upcoming standard, future subjective studies on light field visualization shall benefit from the unified methodologies and from having more comparable results.

\section{EXPERIMENTAL SETUP}

\subsection{Light field displays}

There were 2 real light field displays used in the experiments, namely the HoloVizio 80WLT light field display) 5 (shown on Figure 11) and the HoloVizio C80 light field cinema (shown on Figure 2. The first one is a small, television-like backprojection display with a 30 -inch screen, and the latter is practically a 140 -inch front-projection cinema system. The 80WLT has an angular resolution of 1 degree, and the angular resolution of the C80 is 0.5 degrees. The 80WLT supports content up to a full-horizontal 180-degree FOV, and the FOV of the C80 is 40 degrees. The experiment on the perceptuallysupported viewing distance utilized the 80WLT, and the experiment on the subjectively-preferred viewing distance involved both.

\subsection{Research environment}

Both experiments were carried out in the same laboratory environment, isolated from audiovisual distractions. The brightness values of the 80WLT and the C80 were calibrated to be

\footnotetext{
${ }^{4}$ https://standards.ieee.org/project/3333_1_4.html

${ }^{5} \mathrm{https}: / /$ holografika.com/80wlt/
}

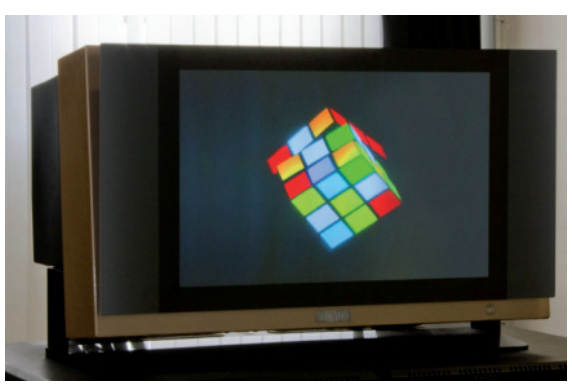

Fig. 1. The HoloVizio 80WLT light field display.

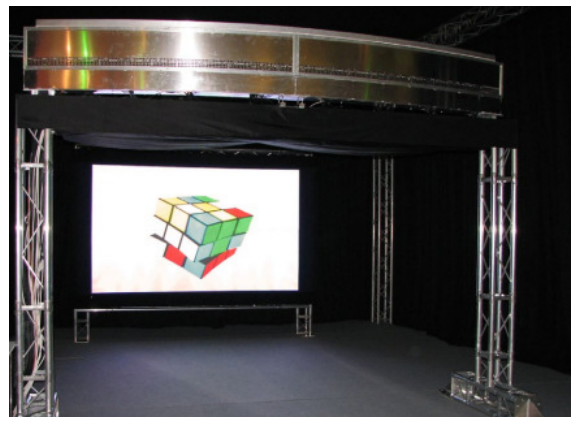

Fig. 2. The HoloVizio C80 light field cinema.

$300 \mathrm{~cd} / \mathrm{m}^{2}$ and $1500 \mathrm{~cd} / \mathrm{m}^{2}$, respectively, and the lighting condition of the laboratory was 20 lux.

Measured from the plane of the screen, the research environment enabled a maximum viewing distance of 8 meters for both displays. As the perceptually-supported viewing distance of the C 80 may be over 8 meters, it was excluded from the appropriate experiment - as already stated at the end of the previous subsection. For the tests on preference, this limitation was taken into consideration during the design phase of the assessment methodology and it is reflected upon in the analysis of the obtained results.

\subsection{Assessment methodology}

For both experiments, the task of the test participants was to determine viewing distances, which were recorded by the conductors of the tests. Along the middle viewing angle, every 25 centimeters on the laboratory floor was visually marked, starting from the plane of the screen and ending at 8 meters. Therefore, there were 32 marks in total, as shown on Figure 3 . Yet, this configuration was only applicable to the 80WLT, because in case of the $\mathrm{C} 80$, the closest viewing distance was limited at 4 meters. This is due to the fact that viewing the screen from a closer position could result in invalid, visualinformation-deprived light field, as the test participants - particularly the taller individuals - could occlude with the rays coming from the optical engines, thus casting a shadow on the screen. Hence, there were only 17 marks in total for the C80, and this altered configuration is shown on Figure 4 


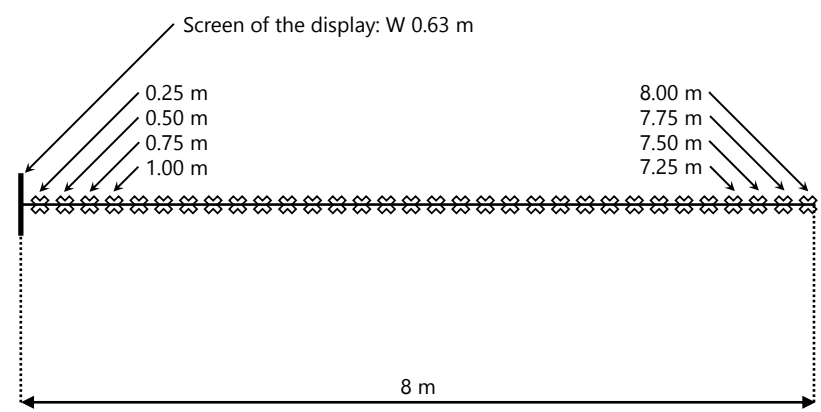

Fig. 3. Viewing positions for the HoloVizio 80WLT light field display.

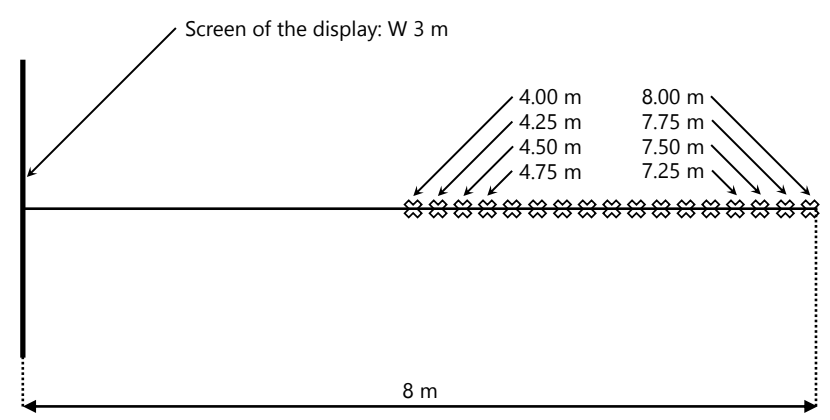

Fig. 4. Viewing positions for the HoloVizio C80 light field cinema.

During the experiment on the perceptually-supported viewing distance, the test participant was asked to stand in front of the display at a comfortably-close distance (i.e., although 0.25 meters was theoretically and also practically an option, the test participant was not asked to view the display from such a short distance), and then to slowly, gradually increase the viewing distance to a point where the perceptual discrimination of content details was no longer possible - the content itself is introduced in the subsequent subsection. The farthest distance at which the visual details were still distinguishable was recorded by the conductors of the experiment.

During the experiment on the subjectively-preferred viewing distance, the test participant was asked to view the visualized content from a multitude of indicated viewing positions, and to find the viewing distance that is personally preferable for the given display and content. The test participant was not instructed to view the content from every single viewing position, the order in which the positions were visited was determined by the test participant, and it was possible to return to positions from which the content had already been observed. When the optimal viewing distance was found (i.e., selected the test participant), it was recorded by the conductors of the experiment.

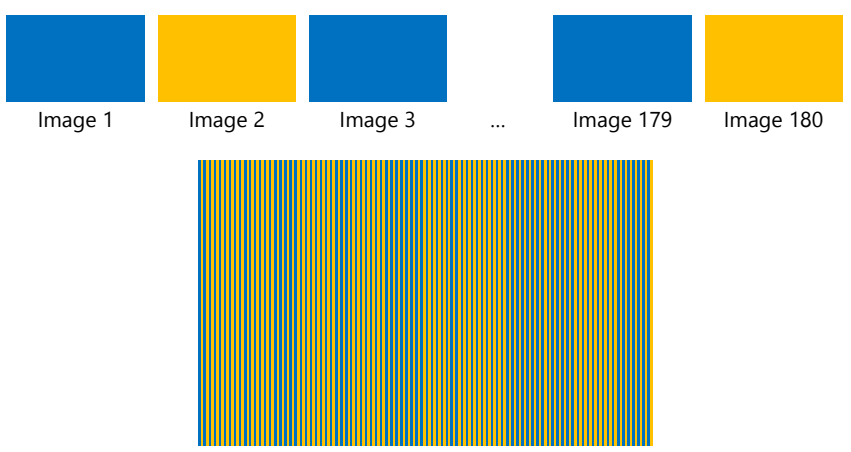

Fig. 5. Concept of the visual stimulus for the experiment on the perceptually-supported viewing distance.

Regarding the assessment itself, in both experiments, the test participant was instructed to stand still during the observation of the content. Natural body sway and head sway was allowed, but for the sake of measurement validity, it was important to remain at a given distance on the line of the middle viewing angle. Furthermore, prior to the experiments, the test participants were screened for normal vision via the Snellen chart (visual acuity) and the Ishihara plates (color vision).

Finally, regarding the distance issue with the C80, the test participants were asked to indicate any potential for the subjectively-preferred viewing distance being beyond 8 meters. This was particularly relevant for test participants who generally preferred greater viewing distances.

\subsection{Visualized contents}

As mentioned earlier, the perceptually-supported viewing distance was addressed via content detail discrimination. For this experiment, we created a visual stimulus that consisted of alternating vertical stripes with high color contrast. Since the 80WLT is a HOP light field display, feeding the converter of the system with an image sequence of alternating plain colors would result in the desired output. As specified in the beginning of this chapter, the angular resolution of the display is 1 degree. This practically corresponds to 1 source view per degree. This is where the FOV becomes relevant, as we need a sequence of 180 alternating images that matches the 2Dequivalent resolution $(1280 \times 768)$ of the display. For colors, we chose blue and yellow. The concept of content generation is shown on Figure 5

For the experiment on the subjectively-preferred viewing distance, we rendered 10 source contents to match the capabilities of the two displays; they were separately rendered for the two displays, each time with the appropriate parameters. Every single content had the same background, which was a medium shade of grey. Content $A$ was a plain-color bust of Aphrodite (laser-scanned model provided by Jotero.com ${ }^{6}$ ).

\footnotetext{
${ }^{6}$ website is no longer available
} 

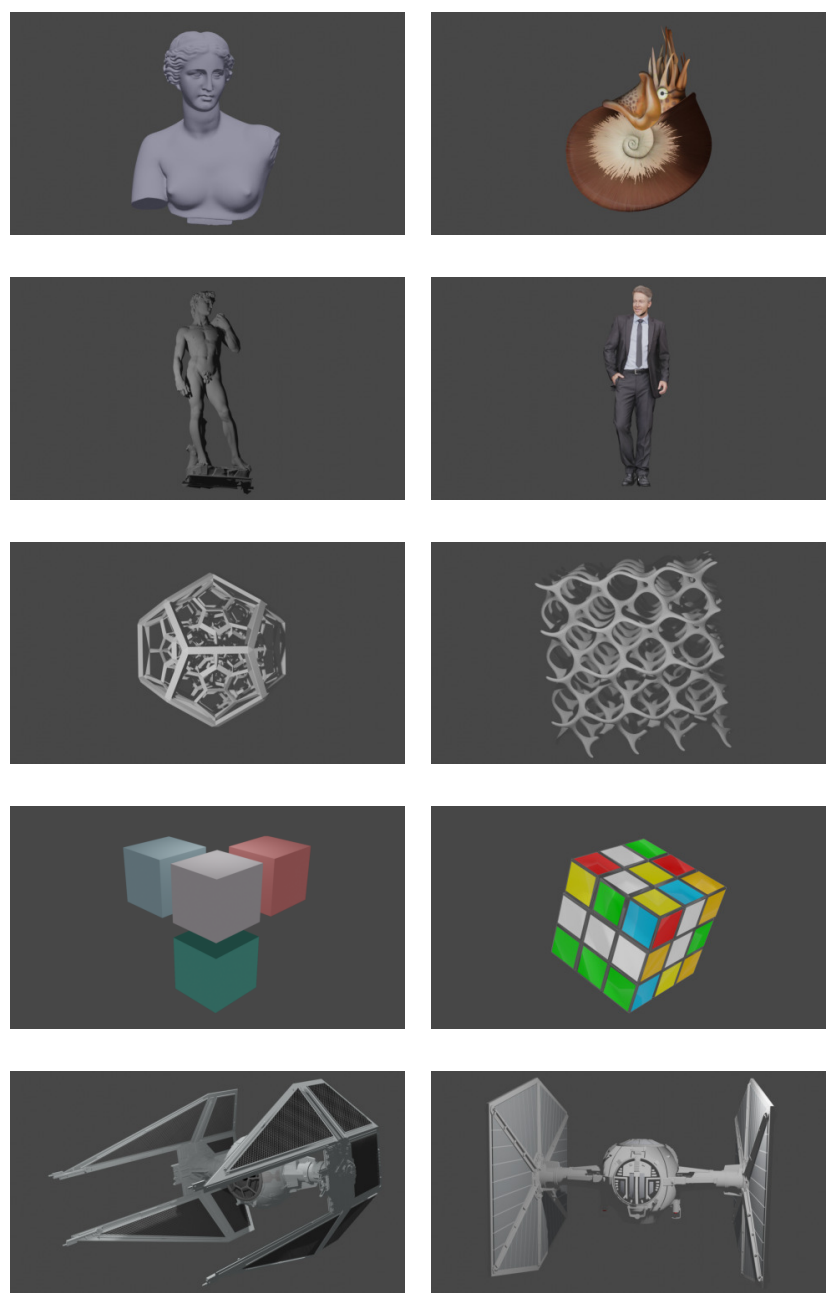

Fig. 6. The source contents of the experiments.

Content $B$ (Ammonite $\mathrm{A}^{7}$ ) had similar spatial dimensions, but it was textured. Contents $C$ (David from The Digital Michelangelo Project ${ }^{8}$ ) and $D$ (Dennis Posed 004 ${ }^{9}$ ) were vertically tall, with the latter being textured. Contents $E$ and $F$ (George W. Hart's Rapid Prototyping ${ }^{10}$ were complex mathematical bodies. Content $G$ was the 3D logo of Holoxica 11 Content $H$ was an animated Rubick's Cube (created by Holografike ${ }^{12}$. Content $I$ and $J$ were the Tie Interceptor ${ }^{13}$ and the Tie Fighter ${ }^{14}$ from the Star Wars ${ }^{\mathrm{TM}}$ franchise. The 10 source contents are shown on Figure 6

\footnotetext{
${ }^{7}$ https://www.turbosquid.com/3d-models/free-ammonite-3dmodel $/ 254206$

${ }^{8}$ https://graphics.stanford.edu/papers/digmich_falletti/

${ }^{9} \mathrm{https}: / /$ free3d.com/3d-model/dennis-posed-004-812878.html

${ }^{10} \mathrm{https} / / / \mathrm{www}$.georgehart.com/rp/rp.html

${ }^{11} \mathrm{https}: / / \mathrm{www} \cdot h$ holoxica.com/

${ }^{12} \mathrm{https} / / /$ holografika.com/

${ }^{13} \mathrm{https}: / /$ downloadfree3d.com/3d-models/aircraft/spaceship/tieinterceptor/

${ }^{14}$ https://downloadfree3d.com/3d-models/aircraft/spaceship/tie-fighterfrom-star-wars/
}

\section{RESULTS}

\subsection{Test participants}

A total of 22 regular test participants completed the experiments (i.e., the subjective tests on the perceptually-supported viewing distance on one device and the subjectively-preferred viewing distance on two devices). 12 test participants were male and 10 were female. The youngest test participant was 21 years old, the oldest was 65, and the average age was 31 .

\subsection{Expert analysis}

As the name suggests, the expert analysis was completed by a set of individuals who are familiar with light field visualization, have extensive knowledge regarding the underlying technology, and thus, perceive the visual quality of such devices differently. As the deviation between the assessment of experts is relatively low, this section reports intervals in which results were gathered.

For the perceptually-supported viewing distance, the obtained results fit into the interval between $4 \mathrm{~m}$ and $5.75 \mathrm{~m}$. Based on Equation 4, the viewing distance threshold for this display is approximately $3.75 \mathrm{~m}$. Therefore, the perceptual limit for the experts is beyond the limit at which the perceived content becomes more 2D than 3D. Additionally, as the height of the display is $390 \mathrm{~mm}, 3.75 \mathrm{~m}, 4 \mathrm{~m}$ and $5.75 \mathrm{~m}$ correspond to $9.62 \mathrm{H}, 10.26 \mathrm{H}$ and $14.74 \mathrm{H}$, respectively.

For the subjectively-preferred viewing distance, results for the HoloVizio 80WLT are shown on Figure 7 As expected, the obtained intervals are rather small. For half of the visualized contents, every expert chose between two distances, and for one (content $E$ ), every expert chose the same distance. For the $\mathrm{C} 80$ - the results of which are shown on Figure 8- this unanimity in the results is even more apparent, as for 8 out of 10 contents, only two different distances were registered.

The reason why there is only minimal deviation within the results registered by the experts is that such individuals tend to rely on the same visual ques of perceived quality; they tend to look for the same things. Hence, their preference is rather based on specific aspects of distance-dependent alterations in perceived quality and their expertise. A much larger deviation and preference variety is expected from the regular test participants.

\subsection{Perceptually-supported viewing distance}

For the 22 test participants, the mean of the registered data regarding the perceptually-supported viewing distance was $5.85 \mathrm{~m}$. However, 6 of them can be considered as outliers, as their natural body sway was greater than what the others had, hence affecting the results. All 6 of them claimed to perceive individual stripes at even $8 \mathrm{~m}$, which was a significant contrast compared to the results of the other test participants. 


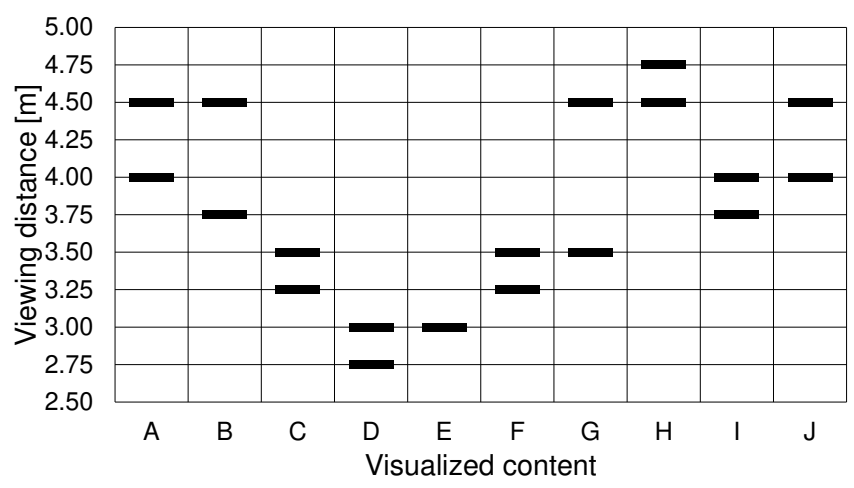

Fig. 7. Results of the expert analysis on the subjectivelypreferred viewing distance via the HoloVizio 80WLT. The markers indicate the intervals used by the experts.

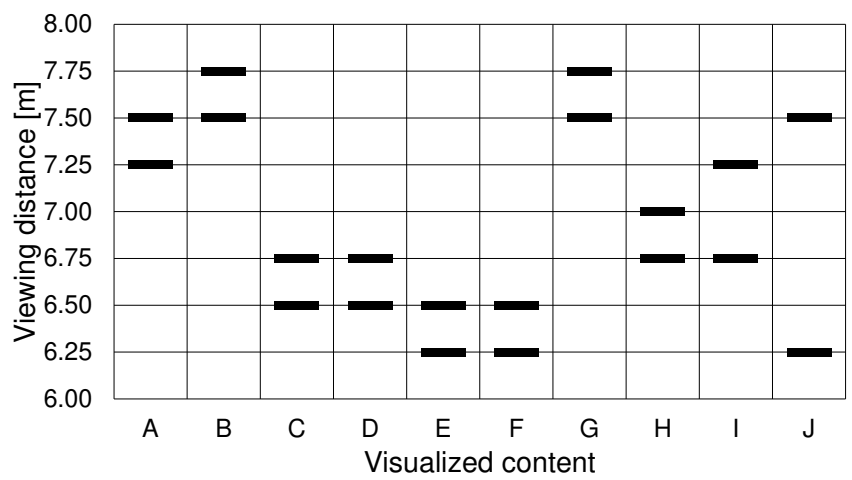

Fig. 8. Results of the expert analysis on the subjectivelypreferred viewing distance via the HoloVizio C80. The markers indicate the intervals used by the experts.

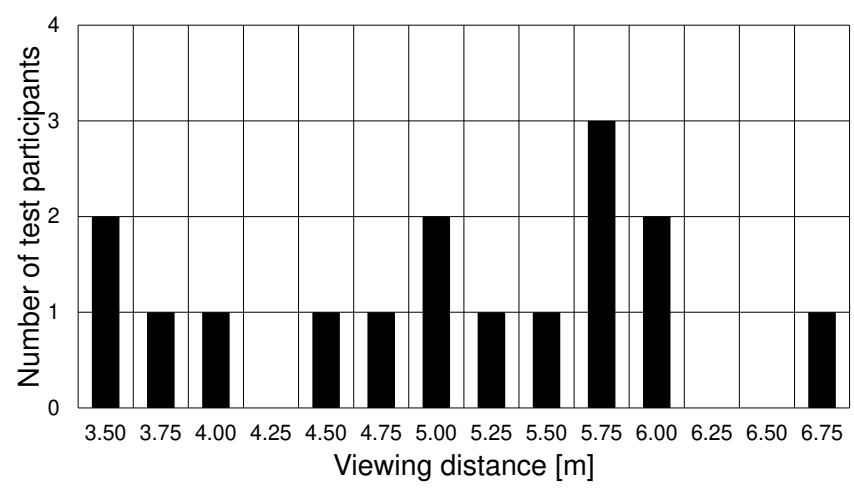

Fig. 9. Results of the subjective tests on the perceptuallysupported viewing distance (excluding outliers).

For the 16 non-outlier test participants, the mean distance was $5.05 \mathrm{~m}$. The distribution of these results are shown on Figure 9. The registered distances are quite evenly distributed within the range from $3.5 \mathrm{~m}$ to $6.75 \mathrm{~m}$. The most frequent

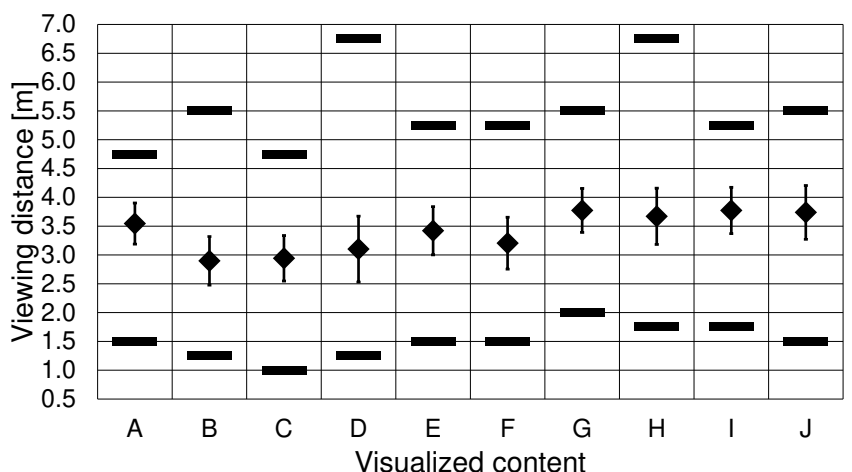

Fig. 10. Results of the subjective tests on the subjectivelypreferred viewing distance via the HoloVizio 80WLT.

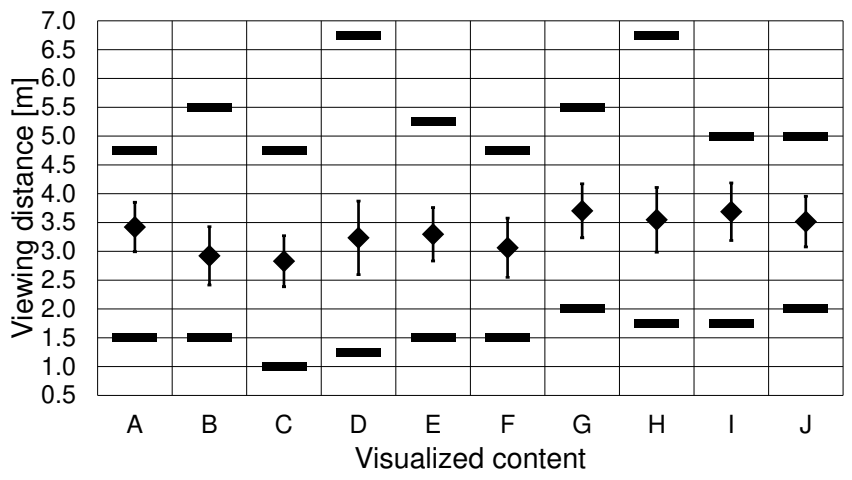

Fig. 11. Results of the subjective tests on the subjectivelypreferred viewing distance via the HoloVizio 80WLT (excluding outliers).

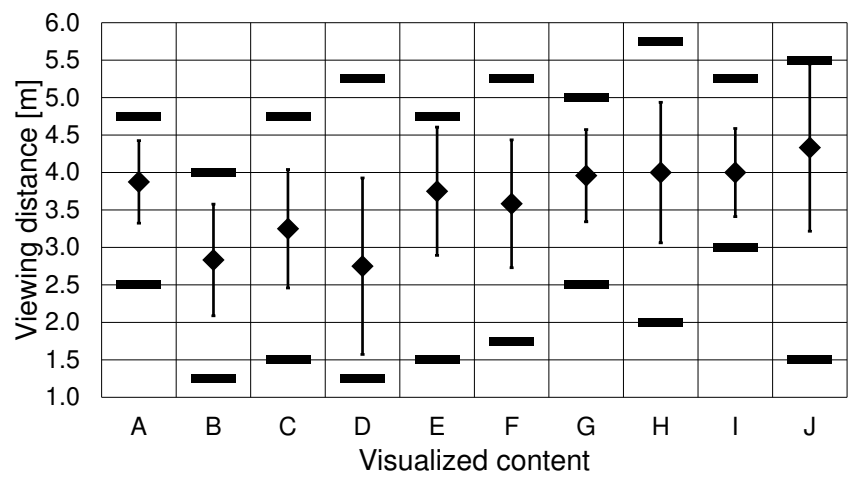

Fig. 12. Results of the subjective tests on the subjectivelypreferred viewing distance via the HoloVizio 80WLT (outliers only).

distance was $5.75 \mathrm{~m}$, which was the top of the interval for the experts. Both results indicate that since Equation 4 is for static observation, the perceptually-supported viewing distance is to be around and above $3.75 \mathrm{~m}$ due to the natural sway. 


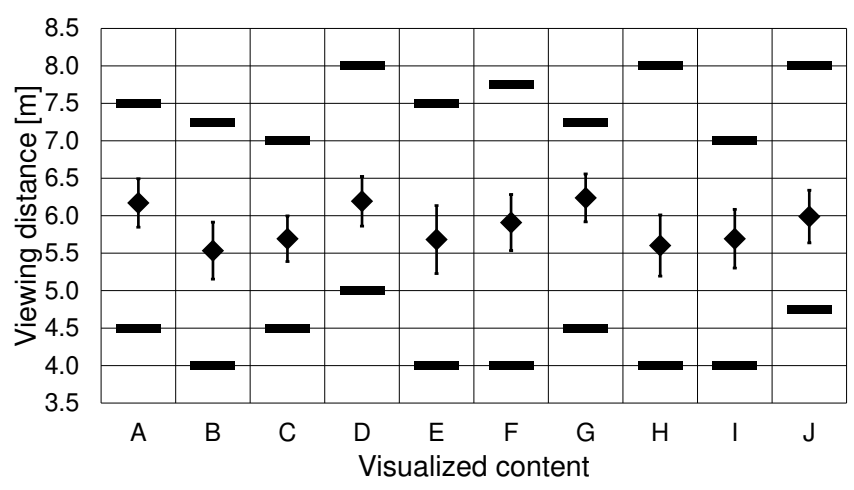

Fig. 13. Results of the subjective tests on the subjectivelypreferred viewing distance via the HoloVizio C80.

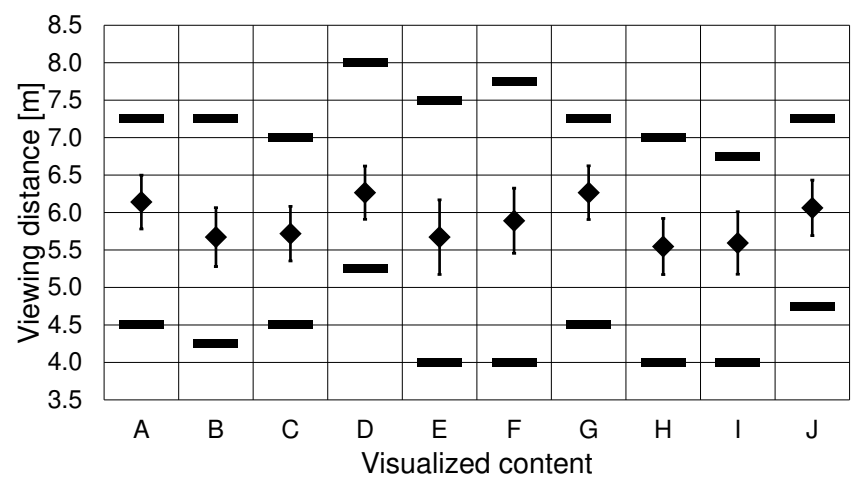

Fig. 14. Results of the subjective tests on the subjectivelypreferred viewing distance via the HoloVizio C80 (excluding outliers).

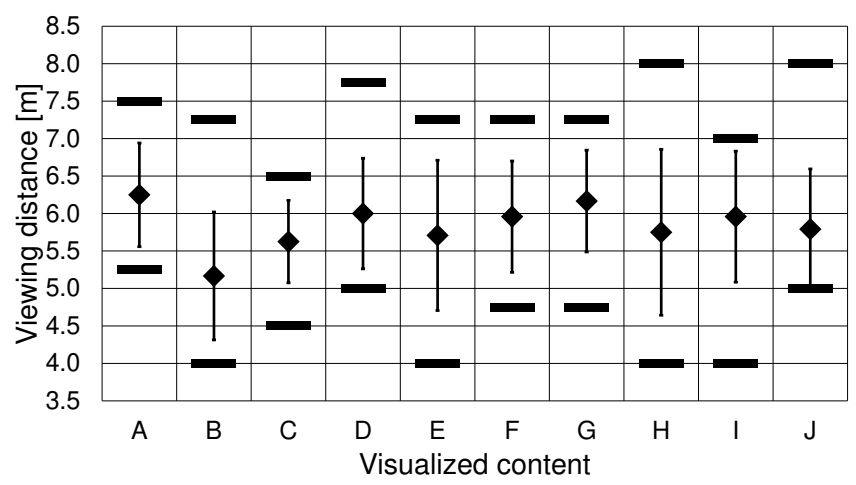

Fig. 15. Results of the subjective tests on the subjectivelypreferred viewing distance via the HoloVizio C80 (outliers only).

Speaking of natural sway, the researchers performing the subjective tests at the laboratory noted that the outliers were typically taller than the other test participants. A greater height may enable larger horizontal disposition at eye level, hence,

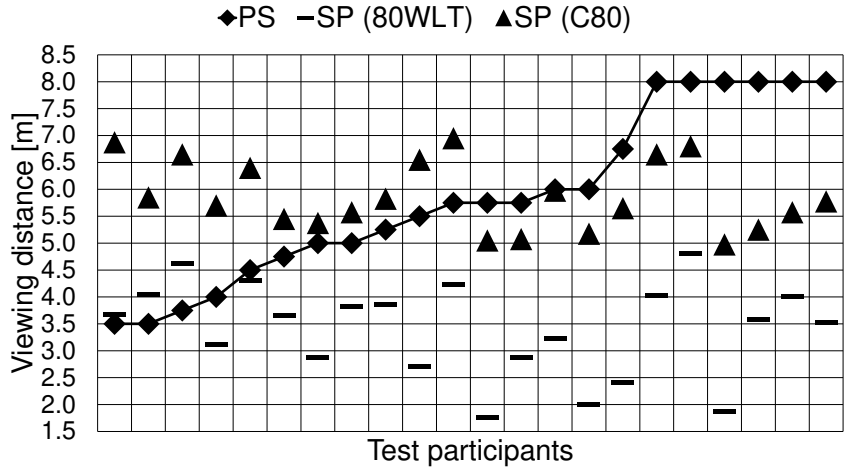

Fig. 16. Results of the subjective tests on the perceptuallysupported (PS) and the subjectively-preferred (SP) viewing distance. Each column represents the mean subjective scores of a test participant, ordered by the results on the perceptually-supported viewing distance.

leading to the particular results obtained for the test at hand. In this context, Equation 4 may be modified as the following:

$$
D_{V}=\frac{D_{E}+D_{S}}{\tan (A R)}
$$

where distance $D_{S}$ is the amount of horizontal disposition caused by sway. As $D_{V}$ is $8 \mathrm{~m}$ in this case, solving this equation for $D_{S}$ gives us $75 \mathrm{~mm}$. Therefore, reaching or passing $75 \mathrm{~mm}$ of sway at a distance of $8 \mathrm{~m}$ may override the perception that would be otherwise normal for the static observation of a light field display with an angular resolution of 1 degree.

\subsection{Subjectively-preferred viewing distance}

Based on our findings regarding outliers, we analyze the obtained results for the subjectively-preferred viewing distance with and without outlier data, and also study the outlier data separately. The results for the HoloVizio 80WLT are shown on Figures 10, 11 and 12, and the results for the HoloVizio C80 are shown on Figures 13,14 and 15 . For each visualized content, the figures provide the mean viewing distance, the 0.95 confidence intervals, and the closest and the farthest preferred distances.

First of all, we conclude that the visualized content does not have a statistically significant impact on the preferred viewing distance. While there are indeed certain contents that were viewed from somewhat closer or farther positions on average, still, their mean values - regardless of test subject classification - fit into relatively small intervals, particularly with respect to the confidence intervals.

The average subjectively-preferred viewing distance for the HoloVizio 80WLT was $3.4 \mathrm{~m}$, with content averages varying between $2.9 \mathrm{~m}$ and $3.77 \mathrm{~m}$. Without the outliers, the average was $3.32 \mathrm{~m}$, and the average for the outliers was $3.63 \mathrm{~m}$. For the C80, these values were $5.87 \mathrm{~m}, 5.53 \mathrm{~m}, 6.24 \mathrm{~m}, 5.88 \mathrm{~m}$ 
and $5.83 \mathrm{~m}$, respectively. In terms of $H$ values, the average subjectively-preferred viewing distances - including all 22 test participants - for the 80WLT ( $0.39 \mathrm{~m}$ screen height) and the $\mathrm{C} 80$ (1.8 $\mathrm{m}$ screen height) correspond to $8.72 \mathrm{H}$ and $3.26 \mathrm{H}$, respectively.

Regarding the experiment on the C80, as stated earlier in the experimental setup, particular attention was assigned to the potential of the subjectively-preferred viewing distance being beyond $8 \mathrm{~m}$. However, no test participants indicated the potential of such personal preference, and the results show that out of the 220 preferred distances, there were only 3 instances when $8 \mathrm{~m}$ was registered, distributed among 3 different test participants. 2 of these test participants were outliers, as seen on Figures 14 and 15

Figure 16 shows the perceptually-supported and the average subjectively-preferred viewing distance for each test participant. As the data is ordered by the perceptually-supported viewing distance, the outliers are situated on the right side of the figure. The comparison shows great diversity with regards to preferences and the lack of clear correlation between the outputs of the experiments. The personal preference average varied between $1.875 \mathrm{~m}(4.8 \mathrm{H})$ and $4.225 \mathrm{~m}(10.83 \mathrm{H})$ for the 80WLT, and between $4.975 \mathrm{~m}(2.76 \mathrm{H})$ and $6.95 \mathrm{~m}(3.86 \mathrm{H})$ for the $\mathrm{C} 80$.

\section{CONCLUSION}

The results of the experiment on the perceptually-supported viewing distance indicate an interval between $4 \mathrm{~m}$ and $5.75 \mathrm{~m}$ for experts, and between $3.5 \mathrm{~m}$ and $6.75 \mathrm{~m}$ for most non-expert test participants, measured on a 30-inch television-like light field display. Registered distances were rather evenly distributed within the interval. Outliers were detected in this experiment, but their subjectively-preferred viewing distance did not deviate significantly from the data of the other test participants. The visualized stimuli did not have a statistically significant effect either, and the subjectively-preferred viewing distance varied greatly. These statements apply to the tests on both light field displays. Further works shall address observer movement in various use cases and the extents of natural sway during forms of static observation.

\section{REFERENCES}

[1] T. Balogh, A. Barsi, P. A. Kara, M. Guindy, A. Simon, and Z. Nagy, "3D light field LED wall," in Digital Optical Technologies. SPIE, 2021.

[2] H. Amirpour, R. Schatz, M. Ghanbari, and C. Timmerer, "On the Impact of Viewing Distance on Perceived Video Quality," in Visual Communications and Image Processing (VCIP). IEEE, 2021.

[3] P. A. Kara, R. R. Tamboli, A. Cserkaszky, A. Barsi, A. Simon, A. Kusz, L. Bokor, and M. G. Martini, "Ob- jective and subjective assessment of binocular disparity for projection-based light field displays," in International Conference on 3D Immersion (IC3D). IEEE, 2019.

[4] P. A. Kara, R. R. Tamboli, O. Doronin, A. Cserkaszky, A. Barsi, Z. Nagy, M. G. Martini, and A. Simon, "The key performance indicators of projection-based light field visualization," Journal of Information Display, vol. 20, no. 2, pp. 81-93, 2019.

[5] A. Ahar, M. Chlipala, T. Birnbaum, W. Zaperty, A. Symeonidou, T. Kozacki, M. Kujawinska, and P. Schelkens, "Suitability analysis of holographic vs light field and 2D displays for subjective quality assessment of Fourier holograms," Optics Express, vol. 28, no. 24, pp. 37 069-37 091, 2020.

[6] A. Cserkaszky, P. A. Kara, A. Barsi, and M. G. Martini, "To Interpolate or not to Interpolate: Subjective Assessment of Interpolation Performance on a Light Field Display," in International Conference on Multimedia and Expo (ICME) 8th Workshop on Hot Topics in 3D Multimedia (Hot3D). IEEE, 2017.

[7] S. Darukumalli, P. A. Kara, A. Barsi, M. G. Martini, T. Balogh, and A. Chehaibi, "Performance comparison of subjective assessment methodologies for light field displays," in International Symposium on Signal Processing and Information Technology (ISSPIT). IEEE, 2016, pp. 28-33.

[8] P. A. Kara, A. Cserkaszky, M. G. Martini, A. Barsi, L. Bokor, and T. Balogh, "Evaluation of the Concept of Dynamic Adaptive Streaming of Light Field Video," IEEE Transactions on Broadcasting, vol. 64, no. 2, pp. 407-421, 2018.

[9] A. Dricot, J. Jung, M. Cagnazzo, B. Pesquet, F. Dufaux, P. T. Kovács, and V. K. Adhikarla, "Subjective evaluation of super multi-view compressed contents on highend light-field 3D displays," Signal Processing: Image Communication, vol. 39, pp. 369-385, 2015.

[10] M. Kawakita, S. Iwasawa, M. Sakai, Y. Haino, M. Sato, and N. Inoue, "3D image quality of 200-inch glassesfree 3D display system," in Stereoscopic Displays and Applications XXIII. SPIE, 2012.

[11] C.-K. Lee, S.-g. Park, S. Moon, J.-Y. Hong, and B. Lee, "Compact multi-projection 3D display system with light-guide projection," Optics Express, vol. 23, no. 22, pp. 28 945-28 959, 2015.

[12] P. A. Kara, A. Barsi, R. R. Tamboli, M. Guindy, M. G. Martini, T. Balogh, and A. Simon, "Recommendations on the viewing distance of light field displays," in Digital Optical Technologies. SPIE, 2021. 